\title{
Dizziness and slow heart rate during exercise
}

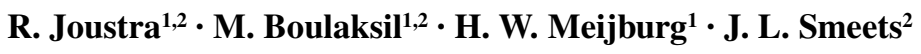

Published online: 11 April 2017

(C) The Author(s) 2017. This article is an open access publication.

\section{Answer}

The ECG at presentation shows an irregular baseline and no $P$-waves, suggesting atrial fibrillation. A regular multiform ventricular bigeminy with fixed coupling interval can be appreciated, which could be typically seen in digitalis intoxication [1].

Other potential ECG features of digitalis intoxication include decreased AV conduction (because of increased vagal effects on the AV node), possibly resulting in high grade $\mathrm{AV}$ block, and enhanced automaticity with frequent premature ventricular complexes. Enhanced automaticity may also induce bidirectional tachycardia with alternating left and right bundle branch block pattern, which is most commonly associated with digitalis toxicity [2].

In our patient, the first complex of the ventricular bigeminy has a left bundle branch block pattern with steep initial ventricular activation, suggesting a focus originating in or near the specific conduction system. The second complex of the bigeminy has an intermediate electrical axis, a left bundle branch block-like morphology, tall R-waves in the inferior leads and a negative QRS-complex in aVL, suggesting a right ventricular outflow tract focus. Although coupling intervals of the bigeminal complexes are fixed, not all RR intervals are at equal length. Therefore, a bidirectional tachycardia is excluded and renders a multifocal origin of the ectopic ventricular complexes most likely.

R. Joustra

r.joustra@jbz.nl

1 Department of Cardiology, Jeroen Bosch Hospital, 's-Hertogenbosch, The Netherlands

2 Department of Cardiology, Radboud University Medical Center, Nijmegen, The Netherlands
A previous ECG (Fig. 1) showed atrial fibrillation and a right bundle branch block. This finding and the fact that no retrograde $P$-waves are seen in between the bigeminal QRS-complexes rules out escape-capture bigeminy as an alternative explanation and favours impulse formation in or near the specific conduction system.

Although our patient had been treated with the lowest daily dose of digoxin $(0.0625 \mathrm{mg}$ q.d.), she nevertheless showed clear signs of digoxin intoxication. This may have been driven by progressive renal failure. Considering the known overlap of serum digoxin levels between groups with and without toxicity [3], we decided not to measure serum levels of digoxin. In addition, no digoxinspecific Fab antibodies were administered since our patient remained haemodynamically stable and she did not develop any other life-threatening complications such as ventricular tachyarrhythmias or bradyarrhythmias.

We decided to implant a two-chamber pacemaker and subsequently reinitiate the beta blocker aiming to optimise rate control, as she developed atrial arrhythmias interspersed with sinus bradycardia (brady-tachy syndrome) one week after discontinuation of digoxin and bisoprolol. Thereafter, she recovered well and was discharged three days after implantation.

Conclusion: multiform ventricular bigeminy with fixed coupling interval caused by digoxin intoxication. 


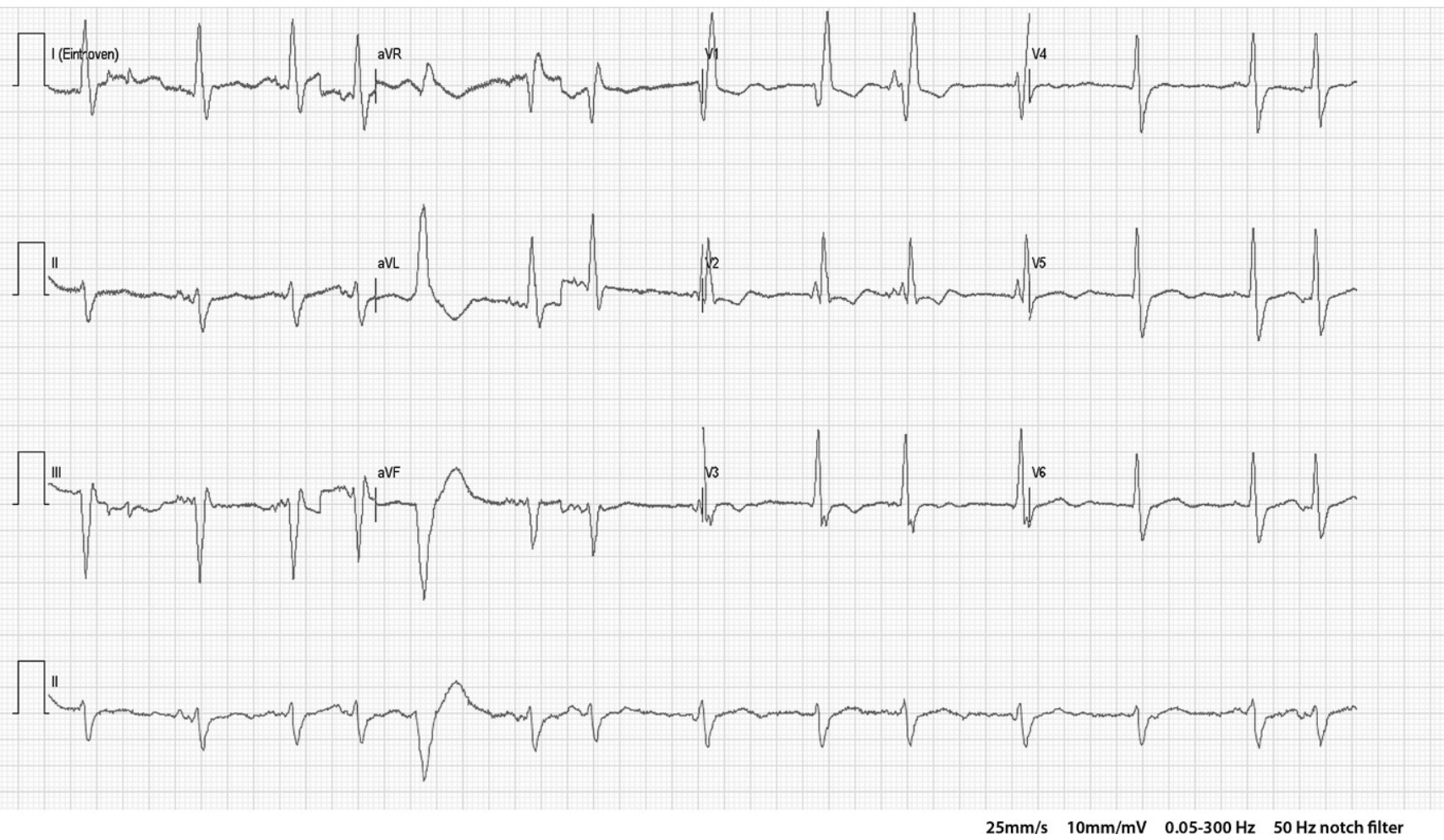

Fig. 1 ECG one month before presentation

Open Access This article is distributed under the terms of the Creative Commons Attribution 4.0 International License (http:// creativecommons.org/licenses/by/4.0/), which permits unrestricted use, distribution, and reproduction in any medium, provided you give appropriate credit to the original author(s) and the source, provide a link to the Creative Commons license, and indicate if changes were made.

\section{References}

1. Wellens H. Digitalis-induced emergencies. In: Wellens H, Conover $\mathrm{M}$, editors. The ECG in emergency decision making. St. Louis: Elsevier; 2006. pp. 170-1.

2. Valent S, Kelly P. Images in clinical medicine. Digoxin-induced bidirectional ventricular tachycardia. N Engl J Med. 1997;336:550.

3. Bayer M. Recognition and management of digitalis intoxication: implications for emergency medicine. Am J Emerg Med. 1991;9:29-32. 